Mitteilungen des Eidg. Institutes für Schnee- und Lawinenforschung

Januar 1966

Nr. 23

Solute Segregation at the Curved Surface of a Growing Crystal (Steady State)

By

C. Jaccard

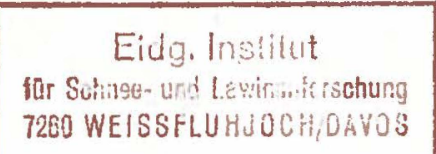

Sonderdruck aus der "Physik der kondensierten Materie"

Band 4, (Schluß-)Heft 5, S.349-354 (1966)

Springer-Verlag, Berlin $\cdot$ Heidelberg $\cdot$ New York

Nicht verkäuflich 



\title{
Solute Segregation at the Curved Surface of a Growing Crystal (Steady State)
}

\author{
C. JACCARD \\ Swiss Federal Institute for Snow and Avalanche Research, WeiBfluhjoch/Davos
}

Received July 2. 1965

We consider a cylindrical crystal growing from the melt at a constant speed with a convex interface. Impurities from the melt segregate at the interface, and their approximate distribution in the solid is calculated in a dimensionless system with the following assumptions: a) the process occurs in the steady state; b) the impurities do not diffuse within the solid; c) the segregation at the interface is large. The determining parameter is the product of growth velocity and average curvature of the interface. When this parameter is large, the impurity concentration near the cylinder mantle is miny orders of magnitude higher than near its axis. This gives rise to a radial refining process, which can be applied when diffusion in the liquid is low and convection has to be prevented. The problem is discussed for different shapes of the interface.

Un crystal cylindrique croît dans la phase liquicic à une vitesse constante avec une interface convexe. Les impuretés du liquide sont rejetées à l'interface, et leur distribution approximative dans le solide est calculée dans un système sans dimension à partir des hypothèses suivantes: a) le processus est à l'état stationnaire: b) les impuretés ne diffusent pas à l'intérieur du solide; c) lia ségrégation à l'interface est suffisamment grande. Le paramètre déterminant est le produit de la vitesse de croissance et de la courbure moyenne de l'interface. Lorsqu'il est grand, la concentration des impuretés sur le manteau du cylindre dépasse de plusieurs ordres de grandeur celle du voisinage de l'axe. Ceci permet un procédé radial de purification qui peut être appliqué lorsque la diffusion dans le liquide est faible et que la convection doit être évitée. Le problène est discuté pour différentes formes de l'interface.

Ein zylindrischer Kristall wächst in der Schmelze mit einer konstanten Geschwindigkeit und besitzt eine konvexe Wachstumsfläche. Verunreinigungen der Schmelze werden an der frenzflïche zurückgestoßen und ihre angenäherte Verteilung in der festen Phase wird unter den folgenden Voraussetzungen berechnet: a) der Vorgang befindet sich im stationären Zustand; b) die Verunreinigungen diffundieren nicht innerhalb der festen Phase; c) die Segregation an der Oberfläche ist genügend groß. Der bestimmende Parameter ist das Produkt der Wachstumsgeschwindigkeit und der mittleren Krümmung der Wachstumsfläche. Wenn er groß ist, ist die Verunreinigungskonzentration am Zy-lindermantel um viele Crößenordnungen höher als bei seiner Achse. Dies ermöglicht ein radiales Reinigungsverfahren, das angewandt werden kann. wenn die Diffusion in der Schmelze klein ist. und wenn keine Konvektion stattfindet. Das Problem wird mit verschiedenen Formen der Wachstumsfläche untersucht.

\section{Introduction}

The segregation of impurities occuring during the growth from the melt of a plane crystal in an infinite straight tube has been investigated from many sides and the phenomenon is now well known $[1,2,3]$. If $s$ denotes the effective segregation coefficient, $D$ the diffusion coefficient of the impurities and $v$ the constant growth velocity, an apparent segregation occurs only at the beginning of the 
growth, corresponding to a length of the order of $D / v s$. In the steady state there is no apparent segregation because the impurities have accumulated near the interface and the concentration has just the right magnitude there to cancel the segregation.

However, these considerations are valid only if the velocity is normal to the interface, i. e. if the crystal surface is plane and normal to the tube axis, otherwise a large radial inhomogeneity of the impurity concentration can be produced. Cylindrical monocrystals of ice that have been grown in an apparatus [5] producing a marked convexity of the interface have most of their impurities concentrated near the mantle: the bulk conductivity has been measured on the original crystals with a diameter of $52 \mathrm{~mm}$ and then after an outer layer of about $3 \mathrm{~mm}$ thickness has been turned down on a lathe; we olsserved a decrease of one order of magnitude between the two measurements. Despite the fact that physical defects may predominate near the mantle, this indicates a considerable accumulation of the impurities in the outer layers. These observations agree with the measurements performed by BLICKS, EGGER and Risul [t], who grew single crystals of ice doped with $\mathrm{HF}$ and $\mathrm{NH}_{4} \mathrm{~F}$ and measured the impurity concentration along the radius. They obtain differences of more than one order of magnitude between the axis and the mantle, which they attribute to the preferred occurence of dislocations and physical defects near the cylinder surface. Their explanation may be only a partial one because their crystals must have grown with a certain convexity; if not they would have obtained polycrystals from fortuitous seeds developing on the vessel surface. The curvature of the interface is always a source of radial inhomogeneities of the impurity concentration, and the purpose of the following analysis is to determine the magnitude of the effect.

\section{The problem}

Let us consider a cylindrical erystal growing from the melt at a constant speed $v$ in an infinite tube. The melt contains only one type of impurity having an asymptotic concentration $c_{0}$. The impurities flow by diffusion in the liquid only and segregate at the interphase according to a coefficient $s$, assumed to be smaller than unity. The shape of the interface has a cylindrical symmetry and because of it the whole process can be expressed in cylindrical coordinates $r$ and $z$.

The impurity concentration $c$ is then governed by the diffusion equation

$$
\frac{\partial c}{\partial t}=D \cdot \Delta c,
$$

where $D$ and $\Delta$ are the diffusion coefficient and the Laplace operator, respectively. If $R$ is the crystal radius, the first two boundary conditions are

$$
\begin{aligned}
& r=R: \frac{\partial c}{\partial r}=0 \\
& z=\infty: c=c_{0} .
\end{aligned}
$$

The third boundary condition at the interphase must express the fact that the rejected impurities are carried away by the diffusion current:

$$
D \operatorname{grad}_{n} c+v_{n}(1-s) c=0 .
$$


Here, $\operatorname{grad}_{n}$ and $v_{n}$ are components normal to the solid surface, which can be expressed by the equation:

$$
z=v t=a R f(r \mid R)
$$

where $f$ describes the shape of the surface, with

$$
f(0)=0 \quad f(1)=-1
$$

and $a$ is a measure of the relative convexity. As we shall be concerned only with the steady state, there is no need for initial conditions.

In order to simplify the problem, we first consider it in a coordinate system moving with the interface at a speed $v$ and we make it dimensionless by introducing the following units: $c_{0}, R$ and $D / R$ for the concentration, the length and the velocity, respectively.

Further, we consider instead of $c$ a "reduced concentration" $g$ equal to $s c$. As the relative concentration in the solid is equal to the apparent segregation coefficient, which we shall call $s^{\prime}(r)$, we obtain this last quantity by just taking the value of $g$ at the interface. Thus the problem reduces to the following system in the steady state:

and

$$
\begin{gathered}
\Delta g+v \frac{\partial g}{\partial z}=0 \\
z=\infty: g=s \\
r=1: \frac{\partial g}{\partial r}=0 \\
z=a f(r): \frac{\partial g}{\partial z}-a \frac{d f}{d r}+v(1-s) g=0
\end{gathered}
$$
form

Elementary solutions of equ. (7) satisfying the conditions (8) and (9) are of the

$$
J_{0}\left(\lambda_{\nu} r\right) e^{-k_{1} z}
$$

where $J_{0}$ are Bessel functions of zero order and the $\lambda_{v}$ are the zeros of the first order functions $\left(\lambda_{0}=0\right)$. The factors $k_{v}$ have the values:

$$
k_{v}=\frac{v}{2}+\sqrt{\frac{v^{2}}{4}+\lambda_{v}^{2}} \quad(\nu=0,1,2, \ldots, \infty) .
$$

We shall restrict ourselves to an asymptotic solution, which has the advantage of its simplicity.

\section{Asymptotic solution for a parabolic interface with a vanishing segregation coefficient}

The simplest shape for a convex (or concave) surface is the parabola, and as it is reasonably well approximated in certain crystal growing devices, we shall calculate the effect for the case

$$
f(r)=-r^{2}
$$


We know further that the apparent segregation is limited by the effective segregation coefficient: the smaller this quantity, the more pronounced are the inhomogeneities. Therefore, it is interesting to learn what can be the maximum effect occuring with an arbitrary small segregation coefficient $s$. In this case (approximation of zero order in $s$ ), equ. (7) has a solution $g_{0}$ depending only of $z$ and satisfying all three conditions independently of the shape function $f(r)$ :

$$
y_{0}=\text { const. } e^{-v z} .
$$

The value of the constant is determined by the condition that the average concentration in the solid at the interface must be equal to unity (as a conscquence of the continuity for the impurity flow), that is :

$$
\int_{0}^{1} g_{0}\left(z=-a r^{2}\right) \cdot 2 r d r=1 .
$$

The expression (15) takes then the exact valuc:

$$
y_{0}=\frac{a v e^{-v z}}{e^{a v}-1}
$$

and we obtain for the radial dependence of the relative concentration in the solicl:

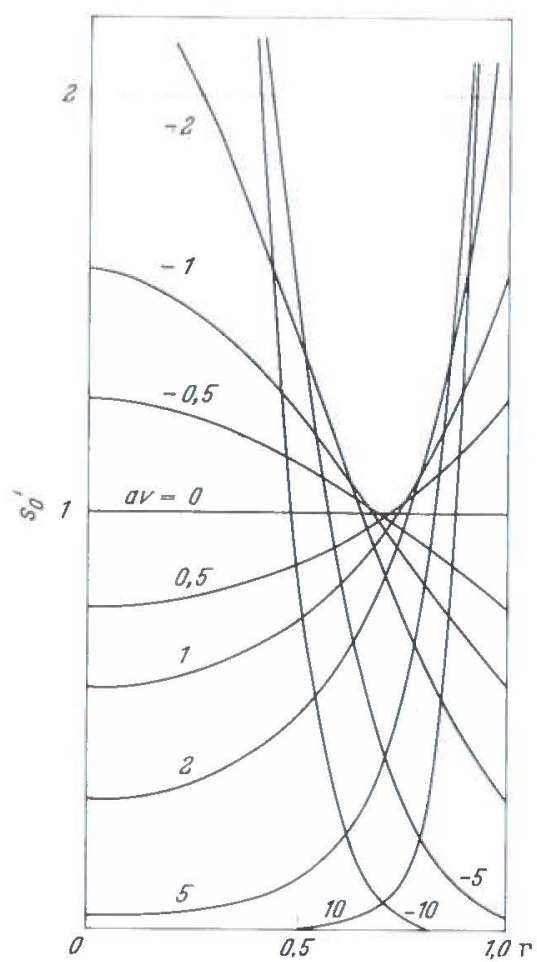

Wig. 1. Distribution of the relative impurity conen. iration in the solicl for a parabolic interfuee as a function of the radius and for diffrent values of the purameter a $r$

$$
s_{0}^{\prime}(v)=\frac{n v e^{a v r^{3}}}{e^{\alpha v-1}} .
$$

This relation is represented in Fig. 1 with the quantity $a v$ as a parameter for convex $(a v>0)$ and concave $(a v<0)$ crystal shapes. The apparent segregation near the cylinder axis $(r=0)$ is very effective as soon as the parameter $a v$ is larger than 5 , and it is compensated by a large concentration increase near the surface $(r=1)$. The magnitude of the effect is gov erned primarily by the two exponential factors in equ. 18, which introduce a very strong dependence on the radius. Values of $a v$ larger than ten are quite possible: the relative convexity $a$ can be made of the order of unity with a suitable device and the velocity unit is proportional to the diffusion coefficient which can be very small (e.g. for ions in water) so that the dimensionless velocity has a large value. If we have for example $v=10^{-6} \mathrm{~m} / \mathrm{s}$, $D=10^{-9} \mathrm{~m}^{2} / \mathrm{s}, R=2 \cdot 10^{-2} \mathrm{~m}$ and $a=0,5$, we obtain a parameter $a v$ equal to 10 , giving the values $s_{00}^{\prime}=4,5 \cdot 10^{-4}$ and $s_{01}^{\prime}=10$ for $r=0$ and $r=1$, respectively. This is a concentration difference of four orders of magnitude between the center and the surface of the cylinder. The absolute difference between $s_{00}^{\prime}$ and $s_{01}^{\prime}$ is equal to $a v$, as it can be seen from equ. 18 . 
When the crystal shape is concave, the effect is reversed, i.e. the higher concentration is near the cylinder axis, but the magnitude of the effect subsists. One sees that $r=1 / \sqrt{2}$ is the limiting radius for an efficient segregation: we always have $s_{0}<1$ for $r<1 / \sqrt{2}$ with a convex shape, and for $r>1 / 1 / 2$ with a concave one.

This approximation of zeroth order can also be calculated easily for any crystal shape, but the constant in equ. 15 is then more complicated to obtain and may have to be computed numerically.

If we consider for example a shape function of the following form:

$$
\begin{aligned}
& 0 \leqq r \leqq r_{1}: f(r)=0 \\
& r_{1} \leqq r \leqq 1: f(r)=-\left(\frac{r-r_{1}}{1-r_{1}}\right)^{2}
\end{aligned}
$$

where the crystal surface is flat within the circle of radius $r_{1}$ and joins the cylinder with a parabola, we obtain according to equ. 15 a concentration distribution with a similar profile. In Fig. 2, these profiles are represented for a parameter $a v=5$ and different values of $r_{1}$. For a convex crystal (full curves) the concentration near the axis is always superior to its value in the purely parabolic case, whereas it is markedly larger at the cylinder sur. face, ranging roughly from $a v$ to $e^{a v}$. For a concave crystal (dashed curves) the opposite is true: near the axis the concentration is appreciably lower but at the surface there is no significant difference. For a radius $r_{1}$ near unity the effect is much larger for a convex than for a concave interface.

These results are strictly valid only if the effective segregation coefficient $s$ is equal to zero, but nevertheless they constitute a good approximation if $s$ is smaller than the minimum of $s_{0}^{\prime}$. In most cases this condition can be fullfilled by a suitable choice of the growth velocity in order to keep the parameter $a v$ in a low value range. If $s$ is greater than the minimum of $s_{0}^{\prime}$, the curves of Fig. 1 then flatten near the value of $s$; this may yield near the axis a value many orders of magnitude larger than $s_{0}^{\prime}$, which is indeed very small for large parameters $a v$. However the

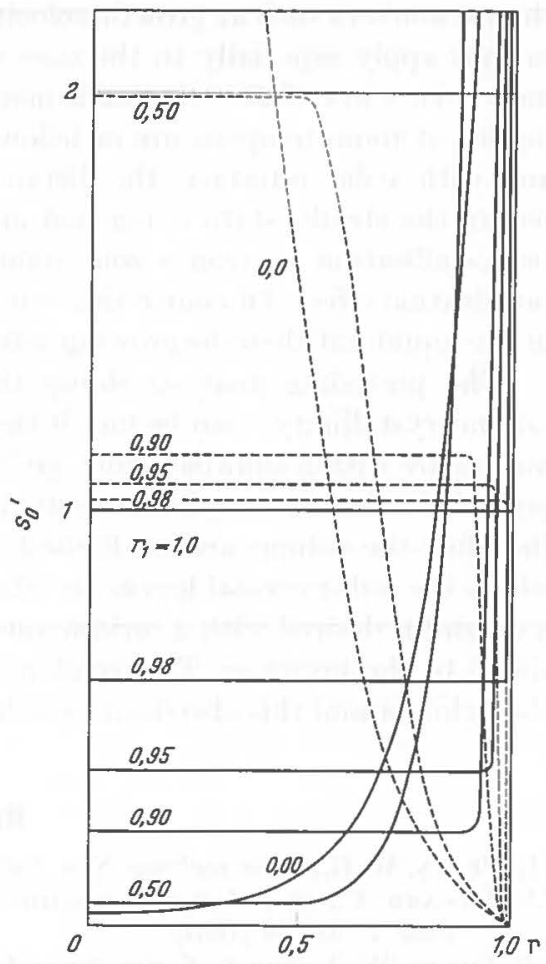

Fig. 2. Distribution of the relative impurity concentration in the solid as a function of the radius for a parameter $a v=5$ and different shapes of the interface (fint for $0<r<r_{1}$ ) high parts of the curves $\left(s_{0}^{\prime}>1\right)$ should not be affected in an appreciable amount as long as $s$ remains much smaller than unity: the absolute value of the difference between the minima of $s^{\prime}$ and $s_{0}^{\prime}$ is negligible and this very small amount of impurities that remain near the axis instead of spreading towards the mantle cannot influence significantly a relative concentration which is larger than unity. 
A general solution, valid for any value of $s$, can be obtained in various ways, e.g. as a series of the elementary solutions (expression 12), the coefficients of which are developed in $s$ and in $a v$. An algorithmic formula allows one to calculate all the unknown quantities, starting from the simple solution known for $a=0$. The term of zeroth order in $s$ corresponds then to the development of equ. 18. However the higher order terms have a very complicated analytical structure so that we will not derive here these cumbersome formulae. The algorithm in principle would be convenient for a digital computer, but as it is itself rather complicated other standard numerical methods for solving partial differential equations might be easier to apply.

\section{Conclusion}

Despite the complexity of an exact solution the asymptotic formula nevertheless gives a description of the phenomenon. This is useful for the design of a crystal growing apparatus because it allows a reasonable selection of the values for the parameters such as growth velocity and shape of the interface. These considerations apply especially to the case where the diffusion of the impurities in the melt is very low. This situation is met with ions and molecules in water or in other liquids at room temperature or below. Even if the segregation coefficient is small and with a flat interface, the distance is small within which purification occurs before the steady state is renched and may be of the order of a few $\mathrm{cm}$. Thus recrystallisation or even a zone refining process is inadequate to obtain a large purifiention effect. Of course this can be greatly improved by a violent convection in the liquid but then the growing solid is no longer a monocrystal.

The preceding analysis shows that the clouble requirement of length and "monocrystallinity" can be met if the interfnce is convex and if the growth parameters are within suitable limits, giving rise to a rudial refining. The refining is not possible in the whole cylinder section but at least in half of it. As the length and therefore the volume are not limited, an efficient repetitive process can be applied where the outer crystal layers are eliminated before ench new recrystallisation. If a crystal is desired with a certain amount of doping, it is convenient to give a flat shape to the interface. This renders the concentration uniform in the middle of the cylinder and this also decreases the apparent segregation.

\section{Refirencess}

[1] Pfann, W. G.: Zone melting, New York: Wiley 1958.

[2] JaCCard, C., et L. Lisvi: Ségrégation d'impuretés dans la glace, Helv. Phys. Acta 12, Fasc. 1, 70-76 (19)(1)).

[3] Tillik, W. A., and R. F. Sererika: Redistribution of solute during phase transformation. J. appl. Plyys. 3is, 2726-2720 (1964).

[4] Blicks, H., H. Ecinen u. N. Rient: Zur Verteilung von Fremdsubstanzen in Eis-Einkristallen. Phys. kondens. Materic 2, 419-422 (1964).

[5] JACCARD, C.: to be published. 